\title{
Co-crystal structure of Protein kinase C-iota with inhibitor reveals an unique binding
}

\section{mode}

Nithya Baburajendran ${ }^{1}$, Jacek Kwiatkowski ${ }^{2}$, Boping Liu ${ }^{1}$, Doris Hui Ying Tee ${ }^{1}$, Thomas H.

$$
\text { Keller }^{1} \text {, Alvin W. Hung }{ }^{1} \text { and Jeffrey Hill }{ }^{1}
$$

1- Experimental Drug Development Centre, A*STAR, 31 Biopolis Way, Singapore

2- Queensland Emory Drug Discovery Initiative, UniQuest, Brisbane, Australia

Protein kinase C (PKC) family of isozymes belong to a family of lipid-dependent serine/threonine kinases and are further subdivided into classical, novel and atypical kinases. PKCs play crucial roles in diverse cellular functions including but not limited to cell proliferation, differentiation and survival. Protein kinase $\mathrm{C}$-iota $(\mathrm{PKC}-1)$ belongs to the atypical subfamily of PKCs and PKC-ı gene (PRK1) is frequently amplified in human cancers. PKC-1 is also overexpressed at the mRNA and protein level in non-small cell lung cancer (NSCLC) and mis-localized in hepatocellular carcinoma (HCC). PKC-1 inhibition in normal cells is well tolerated despite its critical role in cancer cells, thus making PKC- 1 an attractive anti-cancer target.

Our fragment screening identified a pyridine fragment as a weak inhibitor of PKC-1. The potency of the preliminary hit fragment was optimised using structure activity relationship (SAR) and docking studies. One such optimised compound was crystallized with the phosphorylated kinase domain of PKC- 1 and the X-ray structure was solved at $3.2 \AA$. Detailed analysis of the co-crystal structure reveals a distinct binding mode of the fragment involving the post-kinase domain of PKC-1.

Nithya Baburajendran bnithya@eddc.a-star.edu.sg | Jacek Kwiatkowski j.kwiatkowski@uniquest.com.au | Boping Liu bpliu@eddc.a-star.edu.sg | Doris Hui Ying Tee hytee@eddc.a-star.edu.sg | Thomas Keller thkeller@eddc.a-star.edu.sg |

Alvin Hung whung@eddc.a-star.edu.sg|Jeffrey Hill jhill@eddc.a-star.edu.sg 